\title{
Manejo de residuos sólidos mediante la investigación como estrategia pedagógica en la escuela ${ }^{1}$ \\ Solid waste management through research as a pedagogical strategy in school
}

DOI: http://dx.doi.org/10.17981/cultedusoc.9.1.2018.20

Fecha de recepción: 25/05/2018. Fecha de aceptación: 28/08/2018

Eder Enrique Jiménez-Londoño ${ }^{2}$

Rabib del Carmelo Flórez-Romero; Olman Parra-Cristancho; Robinson Zúñiga-Rincones ${ }^{3}$

Para citar este artículo

Jiménez-Londoño, E., Flórez-Romero, R., Parra-Cristancho, O. y Zúñiga-Rincones, R. (2018). Manejo de residuos sólidos mediante la investigación como estrategia pedagógica en la escuela. Cultura. Educación y Sociedad 9(1), 253-264. DOI: http://dx.doi.org/10.17981/cultedusoc.9.1.2018.20

\section{Resumen}

En los últimos años el hombre ha incrementado significativamente la producción de residuos sólidos. Además, la falta de conciencia sobre esta problemática ha limitado la posibilidad de que se consolide una cultura de conservación del medio. El estudio buscó fomentar el manejo de residuos sólidos mediante la investigación como estrategia pedagógica en la escuela. Se guio bajo el enfoque cualitativo, con un diseño de investigación acción participación. Se utilizaron técnicas como diario de campo y observación participante. La unidad de análisis estuvo conformada por ciento veinte (120) estudiantes, de la institución educativa Rodrigo Vives de Andreis de Zona Bananera, Magdalena. Los resultados mostraron que a través del proceso de indagación los estudiantes tomaron consciencia ambiental, lo cual se reflejó mediante charlas sobre contaminación, manejo de residuos sólidos y reciclaje, Además, diseñaron canecas de cartón que distribuyeron en la institución para hacer la recolección y separación de la basura.

Palabras clave: manejo de residuos sólidos, investigación como estrategia pedagógica, escuela.

\begin{abstract}
In recent years, man has significantly increased the production of solid waste. In addition, the lack of awareness of this problem has limited the possibility of consolidating a culture of environmental conservation. The study sought to promote solid waste management through research as a pedagogical strategy in the school. It was guided under the qualitative approach, with a participation action research design. Techniques were used as field diary and participant observation. The analysis unit consisted of one hundred and twenty (120) students from the Rodrigo Vives de Andreis educational institution in Zona Bananera, Magdalena. The results showed that through the process of inquiry the students became aware of the environment, which was reflected through talks on pollution, solid waste management and recycling. In addition, they designed cartons of cardboard that they distributed to the institution to collect and separate them. The trash
\end{abstract}

Keywords: solid waste management, research as a pedagogical strategy, school.

\footnotetext{
${ }^{1}$ Este artículo ha sido derivado del Programa de Fortalecimiento de la Cultura Ciudadana y Democrática CT+I a través de la IEP apoyada en TIC en el Departamento de Magdalena: CICLON

${ }^{2}$ Docente de la IED Rodrigo Vives de Andreis y líder del grupo de investigación Guardianes del ambiente. Correo de correspondencia: amerocu1911@hotmail.com

${ }^{3}$ Docentes de la Institución Educativa Rodrigo Vives de Andreis, Sede Orihueca y miembros del grupo de investigación Guardianes del ambiente.
}

- The author; licensee Universidad de la Costa - CUC. Cultura, Educación y Sociedad vol. 9 no. 2, pp. 253-264. Enero - Junio, 2018 Barranquilla. ISSN 2389-7724 Online 


\section{Introducción}

Resulta oportuno decir que, en las sociedades actuales, los residuos sólidos urbanos llamados usualmente "basura", han tomado una enorme importancia debido a la gran cantidad que se genera a diario y a la diversidad de su composición. Este fenómeno se produce fundamentalmente por la explosión demográfica y la industrialización de los productos de consumo, entre otros aspectos. Casi siempre, al referirnos a la basura, la consideramos algo desagradable, inútil y estorbosa, que nos impulsa a deshacernos inmediatamente de ella sin importarnos si lo hacemos en forma adecuada.

Muchos autores coinciden en que la basura es una mezcla de desecho sólidos que se generan a consecuencia de las actividades humanas, como son las del medio agrícola, industrial, comercial, doméstico y de servicio. En efecto, el manejo impropio y la mala disposición de los residuos sólidos domésticos son problemas comunes que se presentan en cualquier ciudad del mundo. En los países latinoamericanos y en particular el nuestro en Colombia, a lo más que se aspira es depositar los residuos sólidos lejos de los centros de población, con el propósito de reducir los efectos negativos que estos producen al ambiente y a la salud pública.

El fortalecimiento de la educación ambiental en nuestra región está aún en proceso, y constituye un hito histórico, como propuesta académica, social y política de contribución con el fortalecimiento de la educación ambiental. El desarrollo de las actividades humanas antropogénicas, sean simples o complejas, ocasionan, indudablemente, algún tipo de degradación de los recursos del suelo de nuestro planeta, los cuales constitu- yen la base de los procesos productivos, pero, más que nada, de la seguridad alimentaria. Desde la dimensión económica que clasifica a los países, las naciones industrializadas son las que consumen la mayor parte de los recursos naturales de la tierra en beneficio de una pequeña fracción de la población mundial. Estos países marchan casi ciegamente hacia niveles de consumo material y deterioro físico de la naturaleza que a la larga no podrán sostenerse.

Mundialmente se conoce que las problemáticas ambientales van en aumento, por la explotación de los recursos naturales, el uso inadecuado de residuos sólidos, el vertimiento de basura en aguas residuales, la contaminación industrial, entre otras acciones que deterioran el medio, por lo cual es importante diseñar estrategias para preservarlo. Después de la familia, la escuela ha sido la institución encargada de formar individuos competentes para adaptarse a la sociedad y contribuir a ella, por lo cual se deben implementar estrategias desde la educación para generar cambios ambientales significativos que impacten la preservación de los recursos propios de cada región.

La revolución industrial, ciencia y tecnología han generado avances significativos en los últimos años, sin embargo, estos avances también han contribuido a la modificación de hábitos de consumo, remplazando algunos materiales por el sistema de productos desechables, como tarros vasos, cubiertos, platos, frascos plásticos, pañales, botellas, bolsas, entre otros materiales que no son biodegradables, lo cual indica que demoran muchos años en degradarse en el medio, generando graves problemas de contaminación.

Lamentablemente, la institución educativa Rodrigo Vives de Andreis, ubicada 
en el municipio de zona bananera, magdalena, presenta una realidad distinta, y afronta una problemática de contaminación, donde se observa deterioro en las zonas verdes y el paisaje aledaño a la institución, un manejo inadecuado de los residuos sólidos, y la mala disposición de los residuos sólidos domésticos que son problemas comunes a diario en el contexto pertinente. En tal sentido, surge la necesidad de implementar proyectos educativos encaminados a fomentar el manejo de residuos sólidos mediante la investigación como estrategia pedagógica en la escuela.

Esto explicaría las razones, y da origen a problemas que hoy por hoy afecta la comunidad objeto de estudio, producto del deterioro ocasionado por residuos sólidos peligrosos, como el papel usado del baño, pañales desechables, algodón, botellas plásticas, vasos desechables, llanta de autos, electrodomésticos dañados, entre otros, que deterioran y condicionan el ambiente. De igual forma, se debe poner atención al cuidado a esta problemática, ya que, debido a su cantidad, concentración de sus características físicas, químicas, e infecciosas pueden contribuir significativamente en el incremento de la mortalidad o morbilidad de enfermedades serias, irreversibles o causar incapacitación. Además, constituyen un peligro potencial para la salud humana o el medio ambiente cuando se tratan, almacenan, transportan o se disponen inadecuadamente.

Por lo anterior se plantea, opciones de manejo que incluyen esfuerzos de rehúso y reciclaje, tratamientos que involucran compostaje, biogasificación, incineración con recuperación de energía, así como la disposición final en rellenos sanitarios. El punto clave no es cuántas opciones de tratamiento se utilicen, o si se aplican todas al mismo tiempo, sino que sean parte de una estrategia que responda a las necesidades y contextos locales o regionales, así como a los principios básicos de las políticas ambientales en la materia.

Asimismo, mediante la investigación como estrategia pedagógica (IEP) se convierte en una herramienta educativa, que al integrarla al currículo fortalece el proceso enseñanza aprendizaje, partiendo del hecho que las generaciones infantiles y juveniles son nativos digitales, es necesario impartir una formación en ciencia tecnología e innovación, visionando cambios y transformaciones en las dinámicas educativas, de esta manera el maestro a través de la reflexión de su práctica pedagógica puede apoyarse en las pedagogías basadas en investigación, para realizar comunidades de aprendizaje, con la finalidad de generar nuevo saber y conocimiento a través de un aprendizaje en contexto, donde el estudiante logre asociar la teoría a la práctica, propiciando un aprendizaje significativo.

Cabe decir que, la investigación como estrategia pedagógica tiene como eje central de su propuesta la idea que en la sociedad existen saberes propios de una cultura, los cuales negocian permanentemente con las formas establecidas del conocimiento; por ello, la investigación planteada en los grupos infantiles y juveniles buscan la unidad y relación de saber y comprensión como partes complementarias, a través de una propuesta metodológica que realiza el reconocimiento social de los actores, quienes ponen en juego esas diferentes concepciones, que podrán tramitar reconociendo la visibilidad de múltiples métodos investigativos, en coherencia con el tipo de problema. 
Manejo de residuos sólidos mediante la IEP: desde la escuela

En efecto, el acelerado crecimiento urbano y rural del país ha abierto una brecha entre el manejo de los residuos sólidos y su alta producción. En los últimos años con la creación de más productos se ha incrementado la producción de desechos, el volumen de producción de desechos es inversamente igual al nivel de desarrollo de cada país. Debido a esto surge la preocupación de las autoridades e instituciones especializadas en la búsqueda de alternativas concretas para la solución a esta problemática.

Históricamente, el hombre ha depositado sus residuos en torno a sus asentamientos. La complejidad y la diversidad de la actividad humana, a través de la historia, han marcado las pautas y conductas en el manejo y disposición final. Las grandes epidemias y accidentes ocurridos, constituyen el ejemplo más elocuente del alto precio que debe pagar la humanidad por el manejo inadecuado de sus desechos, mediante la integración de la IEP al currículo se busca que los estudiantes identifiquen las diferentes causas, como también los daños y riesgos ocasionados por dicha problemática con la finalidad que diseñen estrategias para dar solución a la misma.

La mayoría de las ciudades latinoamericanas no recolecta la totalidad de los desechos sólidos generados, y sólo una fracción de los desechos recibe una disposición final adecuada, provocando contaminación ambiental y riesgos para la salud humana. (Medina, 2017). El manejo de residuos sólidos (RS) tiene una estrecha relación con la salud de la población, incluyendo, transmisión de enfermedades bacterianas y parasitarias, tanto por agentes patógenos transferidos por los residuos como por vectores que se alimentan y reproducen en los mismos; además, estos incrementan el riesgo de lesiones e infecciones ocasionados por los objetos punzo penetrantes que se encuentran en los residuos, esta condición pone en alto riesgo la salud de las personas que recuperan materiales en los vertederos; donde finalmente la contaminación ocasionada por la quema de residuos, afecta el sistema respiratorio de los individuos, (Sáez, \& Urdaneta, 2014).

Por su parte, Los Residuos Sólidos Urbanos (RSU), se definen como aquellos que son generados por cualquier actividad en los núcleos urbanos, incluyendo tanto los de carácter doméstico como los provenientes de cualquier otra actividad generadora de residuos dentro del ámbito urbano, es decir, son aquellos que se originan en los núcleos de población como consecuencia de la actividad habitual y diaria del ser humano, (García, Toyo, Acosta, Rodríguez, \& El Zauahre, 2015).

Según la UNESCO, los RSU de acuerdo a su composición pueden ser orgánicos (biodegradables), los cuales son descompuestos por la acción natural de organismos vivos como lombrices, hongos y bacterias principalmente, o inorgánicos (no biodegradables) cuyas características químicas sufren una descomposición natural muy lenta. Muchos de ellos son de origen natural pero no son biodegradables, por ejemplo, el vidrio. Generalmente se reciclan a través de métodos artificiales y mecánicos, como las latas, envases de vidrio, plástico y gomas.

El problema de los RSU tiende a agravarse como consecuencia del acelerado crecimiento de la población y la concentración en las áreas urbanas, cambios de hábitos de consumo (status social) y otros factores que producen contaminación del ambiente con el consecuente deterioro de 
los recursos naturales. Asimismo, el desarrollo económico, la industrialización y la implantación de modelos económicos que conllevan al aumento sostenido del consumo, han impactado significativamente en el volumen y la composición de los residuos producidos por las sociedades del mundo.

Quiñónez Cortez, L (2016), menciona que la generación de residuos sólidos urbanos ha registrado incrementos cuantiosos, lo cual se ha convertido por sus características, en un problema para las localidades poblacionales. Dicha situación se encuentra dada principalmente por la existencia de un manejo inadecuado de los desechos, así como por la carencia de programas de educación ambiental y la falta de conciencia del ser humano sobre el tema. Aspectos como la demografía, el modo consumista de los seres humanos y las actividades cotidianas de la dinámica poblacional, son unas de las principales causas que contribuyen al desarrollo de este problema y a las disímiles consecuencias socio ambiental que repercuten sobre el paisajismo local, la propagación de enfermedades y contaminación frecuente a causa de la generación de desechos sólidos.

Según Moyano (2012), los residuos se pueden clasificar en cinco categorías; residuos domésticos, los cuales son producidos por las actividades del hogar, como la basura generada al cocinar, los envases de elementos de aseo, los vertimientos de aguas residuales, entre otros. Residuos comerciales; son todos los desechos generados por los locales comerciales, como restos de comida, frutas, carne y otros elementos en descomposición que generan contaminación. Residuos agrícolas o forestales; estos son producidos por la tala de árboles o quema de los mismos. Residuos ganaderos; como la materia fecal de los animales. Y finalmente los residuos industriales; estos son cenizas, gases tóxicos, plásticos, humo, sustancias químicas, carbón, vidrio, madera o escombros.

La educación ambiental tiene un papel importante en el manejo de residuos sólidos por parte de los habitantes de una comunidad, en ese sentido, estos deben identificar a través de instrumentos de medición, para caracterizar la problemática, los impactos y las causas que la generan, para posteriormente sensibilizar, educar, capacitar y crear estrategias de intervención que involucren a los responsables generando espacios de gestión para la comunidad, (Sandoval, \& Barrera, 2017).

En ese sentido, la educación ambiental es un proceso educativo planificado que busca suministrar instrucciones basadas en los más recientes datos científicos, al igual que en el sentimiento público prevaleciente diseñado para apoyar el desarrollo de actitudes, opiniones y creencias que apoyen a su vez la adopción sostenida de conductas que minimicen lo más que sea posible la degradación del ambiente original o las características geológicas de una región, la contaminación del agua, aire o suelo, y las amenazas a la supervivencia de otras especies de plantas y animales, de esta manera la educación ambiental busca propiciar la adquisición de conocimientos para la comprensión de la estructura del medio ambiente, suscitando comportamientos y actitudes que hagan compatibles la mejora de las condiciones de vida con el respeto y la conservación del medio desde un punto de vista de solidaridad global para los seres humanos, (Carrato \& Marval, 2007).

Según Herrera \& Bravo, (2013) es necesario tener en cuenta desde la economía ecológica el proceso denominado 
"Metabolismo social", el cual, indica que la sociedad necesita de unos recursos naturales de los que se apropia con el fin de transformarlos, hacerlos circula y a partir del uso generar desechos que son excretados a la naturaleza, lo cual se puede observan en el diseño o elaboración de las huertas escolares ecológicas, donde los estudiantes logran tomar unos recursos $\mathrm{y}$ transformarlos, generando un beneficio de consumo a la comunidad.

La educación a lo largo del tiempo ha presentado cambios significativos, que invitan al docente a diseñar e implementar nuevas estrategias pedagógicas para dirigir el proceso de enseñanza con sus estudiantes, una estrategia pedagógica es un conjunto de actividades que se realizan con un orden lógico y coherente en función del cumplimiento de objetivos del plan académico. Es decir, es una planificación que contiene métodos o acciones que permiten al estudiante alcanzar los logros propuestos, mejorar su aprendizaje y facilitar su crecimiento personal, (Picardo, Balmore, \& Escobar, 2004).

Partiendo de lo anterior, cuando la cultura de una organización en este caso la escuela, está alineada con sus valores, atrae a aquellos que se sienten identificados con ésta, lo que, ayuda a la institución a estimular a sus estudiantes y lograr un alto nivel de compromiso, lo ideal es trabajar incesantemente en fortalecer la cultura de la organización de acuerdo a los principios corporativos, que deben ser compartidos y construidos con los estudiantes. (Ramírez, R. 2018).

En la actualidad, existen propuestas metodológicas de enfoques basados en investigación, entendidos como la forma de materializar lo que se hace y como se implementa donde surgen infinidad de propuestas metodológicas que se basan en un enfoque o toman varios para ha- cer de ellos una propuesta metodológica; es el caso de las pedagogías basadas o fundadas en investigación, las cuales se desarrollan en diferentes contextos y asumen variados caminos en coherencia con los paradigmas y corrientes en los cuales se inscriben. Dentro de esta teoría basada en investigación se encuentra la Enseñanza por descubrimiento y el Programa Ondas, con su propuesta de la Investigación como Estrategia Pedagógica (IEP), como una perspectiva que se desarrolla en ese enfoque y toma elementos de la pedagogía critico-liberadora y del enfoque socio cultural, (Mejía y Manjarrés, 2010).

Los contenidos del proceso educativo se convierten en problemas, y a partir de preguntas se estructura el currículo, en donde los avances y desarrollos de los estudiantes se determinan por la ampliación de estructura previa que se manifiesta en lenguaje, raciocinios y conocimientos. Ese descubrimiento no es necesariamente autónomo, sino que se hace guiado por el profesor, quien se encarga de planificar los ejercicios y los procedimientos para el fin buscado, (Pozo, J. \& Gómez, M, 2006).

Por ello, la propuesta no es formar científicos, es construir una cultura ciudadana y democrática en ciencia, tecnología e innovación para estos tiempos de un mundo construido sobre el conocimiento, la tecnología, la información y la comunicación. Este ejercicio pedagógico aparece como fundamento de una nueva forma de lo público que, a través de la idea de justicia educativa y justicia curricular, trabaja por construir sociedades más justas $\mathrm{y}$ menos desiguales. $\mathrm{Y}$ si después de esto los niños, niñas y jóvenes optan por ser científicos lo serán también de otra manera, como parte de la búsqueda iniciada desde la propuesta metodológica que contiene como valores fundamentales esas 
capacidades de lo humano (cognitivas, afectivas, valorativas y de acción) sobre las cuales se fundamenta la investigación como estrategia pedagógica.

En ese sentido, pensar la investigación desde las corrientes educativas criticas significa una lucha teórico-práctica por la manera como sus presupuestos sobre el conocimiento, la ciencia, su epistemología, la cultura, lo humano y los grupos sociales enmarcan una acción que durante cuatrocientos años ha sido señalada como objetiva, y que no solo ha construido una forma de ella, sino que también ha ayudado a generar formas de poder que en la sociedad han servido para el control y la gestación de desigualdades, y en estos tiempos, nuevas formas de acumulación y dominación (Ortega P, Peñuela D \& López D, 2009).

Cochran y Litle (2006), plantearon la existencia de conocimientos y reflexiones en la acción, lo que permite integrar en las actuaciones explícitas e implícitas lo cognitivo, lo emocional, la teoría y la práctica. Reconociendo que tanto quien enseña como quien aprende dentro de una comunidad trabajan para generar conocimiento local, prever su práctica y teorizar sobre ella, interpretando las conclusiones de otros, todo lo anterior es posible al integrar la investigación al aula.

Cabe decir que, se requiere mostrarse de acuerdo la importancia de la investigación en la sociedad, a la vez que se develan los intereses presentes. En su práctica forja una mirada crítica, que en coherencia con los tiempos presentes se ve obligada no solo a la acusación, sino también a apropiarse de ella como una realidad muy importante en esta época para construir propuestas que a la vez que promuevan su apropiación práctica crítica, sirva no solo para modernizar, sino, ante todo, para transformar contex- tos, culturas, epistemologías, y redirigir sus escenarios de poder para construir subjetividades y ciudadanías que reconociendo el lugar de lo local se abran a un tiempo-espacio global y a las nuevas mediaciones científico-tecnológicas de este tiempo para hacer concreta la construcción sociedades más democráticas, justas y humanas, así como la necesidad de un planteamiento sobre ella en cualquier proyecto emancipador.

\section{Metodología}

\section{Diseño}

El estudio se guio bajo el enfoque cualitativo, con un tipo Investigación por acción Participación (IAP). La investigación cualitativa busca conocer e interpretar la realidad de los participantes a través de sus propias experiencias, entregando una información subjetiva del fenómeno de estudio. (Hernández, Fernández, y Baptista, 2010). En la IAP el conocimiento de la realidad no se descubre ni se posee es el resultado de la trasformación subjetiva y objetiva de el mismo proceso de investigación, en consideración a esta la validez del conocimiento se da por la capacidad que posee para orientar la trasformación de una comunidad o grupo mejorando la calidad de vida de sus integrantes.

La IAP es un proceso educativo de autoformación en el cual las personas que pertenecen al grupo sobre quienes esta la realidad estudiada, tienen una participación directa en el proceso de investigación y en la producción de conocimiento de su realidad. En cuanto a los instrumentos de recolección se utilizó el diario de campo, este es un instrumento que ayuda al investigador a inmiscuirse de forma total en el ambiente con el cual se va a encontrar, es un proceso de hechos, recolección de datos y análisis. 


\section{Participantes}

En cuanto a la población sujeto de estudio, la Institución Educativa Rodrigo Vives de Andreis se encuentra ubicada en el corregimiento de Orihueca, Zona Bananera del departamento del Magdalena. Cuenta aproximadamente con 2400 estudiantes repartidos en cinco sedes ofreciendo el servicio educativo en preescolar, básica primaria, básica secundaria y media técnica, además cuenta con el programa de educación para adultos en la jornada nocturna. La muestra estuvo conformada por ciento veinte (120) estudiantes, con edades que oscilaron entre los trece (13) y dieciséis (16) años, de los grados octavo, noveno y décimo de bachillerato, de la sede Orihueca, el nivel socio-económico de la región donde se encuentra la institución es estrato 1, donde las actividades productivas de la población están basadas en la agroindustria teniendo como base fundamental en cultivo del banano.

\section{Técnicas e instrumentos}

El investigador entiende a los participantes y no se dedica únicamente a registrar hechos. El diario de campo es parte de un registro cronológico que se desarrolla mediante la sesión compartida con los infantes, es donde se describe detalladamente los aspectos positivos como negativos colocando significado a cada uno de ellos como un recurso de mejoras en todas las áreas con el grupo a trabajar considerando los recursos manejados y utilizados. Un cuaderno donde se va a descubrir la manera de expresar y escribir frente a la relación que se tiene con los infantes, es un momento de no cohibir lo vivido y las experiencias obtenidas.

\section{Procedimiento}

La metodología del proyecto de investigación se realizó a partir de los recorridos de las trayectorias de indagación, basados en la integración de la IEP apoyada en TIC al aula, articulado al plan de estudios, desde el área de sociales se estructuraron temáticas relacionadas con la violencia sexual y estrategias preventivas del cuidado del cuerpo, lo anterior con la finalidad de generar una trasformación social y educativa a través del aprendizaje en contexto, involucrando intereses, motivaciones y realidades de los estudiantes para generar conocimiento científico, donde se establecieron cinco (5) momentos que especifican la ejecución y las metas trazadas para darle solución a la pregunta problema planteada. Los momentos utilizados se presentan a continuación.

- Momento (1): selección y análisis de la población sujeto de estudio.

- Momento (2): integración de la temática mediante la IEP al aula de clases, se integraron grupos estudiantiles para seleccionar problemáticas relacionadas con el manejo de residuos sólidos y la contaminación ambiental, los estudiantes se plantearon preguntas y con el acompañamiento del docente realizaron el proceso de indagación mediante herramientas virtuales y entrevistas a la comunidad.

- Momento (3): diseño de estrategias de intervención por parte de los estudiantes para dar solución al problema planteado.

- Momento (4): análisis de los resultados de la implementación de la estrategia a través de la observación y diario de campo.

- Momento (5): propagación y divulgación de los resultados. 


\section{Resultados}

A continuación, se presentan los principales hallazgos de la investigación realizada, donde se evidencio que a partir el fomento del manejo de residuos sólidos mediante la investigación como estrategia pedagógica, los estudiantes tomaron consciencia ambiental partiendo del proceso de indagación en bases de datos abiertas y la plataforma de google académico, donde revisaron diferentes artículos de trabajos realizados frente al tema, seguidamente diseñaron y aplicaron entrevistas a la comunidad sobre sus actitudes ambientales, encontrando que en el municipio de Zona bananera existe una problemática de contaminación fuerte debido al manejo inadecuado de los residuos sólidos urbanos, donde observaron que la comunidad tiene poco conocimiento del daño ambiental que están produciendo.

Las principales fuentes de contaminación encontradas por los estudiantes fueron las actividades domésticas, arrojando basura en lotes vacíos, lavando la ropa en el rio con detergentes, dirigiendo vertimientos de aguas residuales y desechos humanos a este, quemando basura, entre otras actividades que dañan el medio, frente a esta situación los jóvenes crearon estrategias pro-ambientales dirigidas por ellos, mediante charlas sobre la contaminación, manejo de residuos sólidos y reciclaje, realizaron una jornada de recolección de basura y separación de los materiales que se podían reciclar.

Además, en la institución los estudiantes realizan actividades en contra de las instalaciones el inmobiliario, paredes, áreas comunes donde ellos se forman, uso inadecuado del baño y mal manejo de las basuras, por lo cual diseñaron huertas, donde hicieron jornadas de ornamentación con plantas florales, diseñaron canecas de cartón que distribuyeron en la institución para hacer la recolección y separación de la basura, dictaron charlas de reciclaje, orientadas a generar consciencia institucional.

A través de la observación registrada en el diario de campo, los docentes lograron identificar mediante la integración de la IEP al currículo, los estudiantes presentaron motivación y la dinámica de las clases fue totalmente interactiva, con interés desde el proceso de indagación en bases de datos, hasta la apropiación del conocimiento para realizar carteles, organizar charlas y diseñar estrategias para aportar de manera positiva a la generación de un cambio social en la comunidad.

En dicha actividad se logró capacitar directamente a las personas de la institución, a través de charlas sobre minimización de generación de residuos, separación de residuos en la fuente, reúso y reciclaje de residuos. En estas charlas se facilitaron folletos específicos del tema y se enfatizará la importancia de los habitantes en la etapa de generación, manipulación y procesamiento en el origen de los residuos sólidos y como el personal de la institución puede colaborar disponiendo adecuadamente sus residuos dentro de los recipientes de colores según el tipo de residuo y se complementará la sensibilización ambiental con la fijación de afiches en lugares públicos para recordar a los habitantes el manejo adecuado que le deben dar a los residuos sólidos.

Finalmente, se realizó la propagación de la información, donde el grupo divulgó con la comunidad educativa el proceso y los resultados de la investigación, con el propósito de compartir los beneficios y alcances de la misma, para que éstos puedan ser útiles a la colectividad, los grupos deben compartir sus resultados en eventos, a través de diferentes medios de comunicación. Los productos de la investigación se divulgan en los espacios 
virtuales y físicos del programa Ciclón consolidando la comunidad de práctica saber, aprendizaje, conocimiento y trasformación: Espacios físicos: asesorías de línea temática, eventos de apropiación: ferias, encuentros el maestro tiene la palabra, comités subregionales, espacios de formación de maestros y publicaciones impresas.

En el caso de la IEP, se parte de la pregunta del sentido común de los niños, niñas y jóvenes para reelaborarla en la perspectiva de la educación popular, que reconoce la existencia de saberes comunes y elaborados así en la negociación con los conocimientos disciplinares, y de las maneras como se correlacionan en la negociación cultural, realizando un aporte social a las problemáticas propias donde el estudiante se desenvuelve, aportando a un aprendizaje en contexto. (Mariño, 2010).

En otras palabras, el concepto de manejo integral de los Residuos sólidos le da un nuevo espacio al enfoque comúnmente conocido como la jerarquía del manejo de residuos sólidos, el cual prioriza las opciones de manejo de residuos en un orden de preferencia que parte de la prevención en la generación, del rehúso, reciclaje o compostaje, de la incineración con recuperación de energía, de la incineración sin recuperación de energía, y del confinamiento en rellenos sanitarios como última opción. Este enfoque ha influido significativamente en las decisiones y estrategias de manejo de residuos a nivel local, nacional e internacional durante los últimos años.

No siempre el reciclado de residuos es la mejor opción desde la perspectiva ambiental y económica, como lo muestra la aplicación del análisis del ciclo de vida comparativo, en el que se pone en perspectiva esta opción respecto de la generación de los materiales primarios correspondientes. La selección de las combinaciones de formas de manejo de los residuos y de las prioridades que deben asignárseles, requiere hacerse con base en diagnósticos que permitan conocer las situaciones que privan en cada localidad respecto del tipo y volúmenes de residuos que se generan, la infraestructura disponible o accesible para su manejo y los mercados de los materiales secundarios, entre otros.

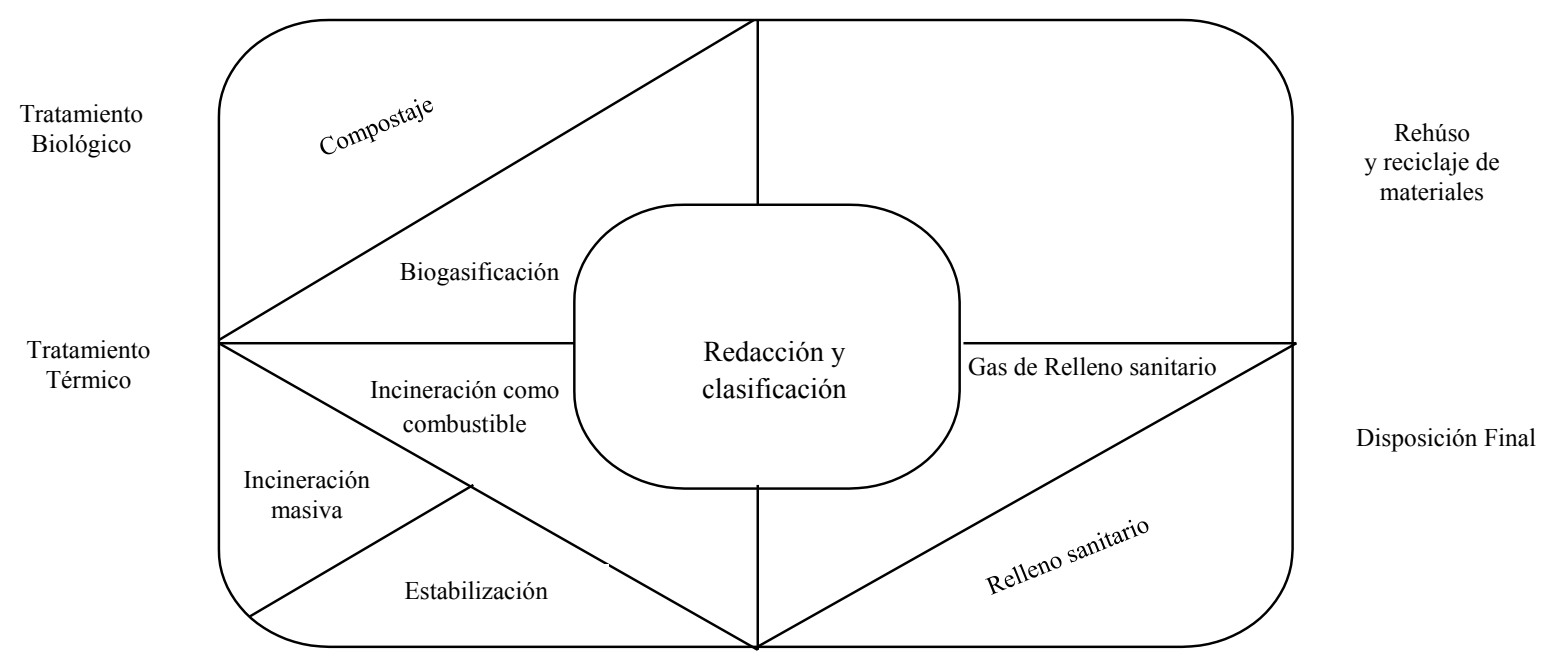

Figura 1. Manejo integral y Sustentable de los Residuos Sólidos (Garmendia, A, Dávila, A. 2006). Fuente: elaboración propia (20018). 


\section{Conclusiones}

En fin, la medida en que los residuos contienen sustancias, organismos patógenos y elementos orgánicos que pueden dar lugar entre otros problemas a la contaminación del ambiente, así como a la exposición humana y terrestre a agentes que les pueden dañar, representan un riesgo que es preciso evaluar, prevenir y controlar. Los riesgos en el manejo integral de los residuos pueden darse desde el lugar en el cual se generan, donde se acopian, durante la recolección y el transporte y en las instalaciones en las cuales se les someterá a tratamiento o disposición final; por ello es preciso identificar y caracterizar los riesgos particulares en cada fase a fin de plantear medidas para prevenirlos o reducirlos. Al mismo tiempo, es preciso definir qué es lo que se busca proteger, ya sea seres humanos (trabajadores y población en general), organismos, recursos naturales, cuerpos de agua, suelos, calidad del aire $\mathrm{y}$ bienes materiales o culturales.

El manejo de residuos sólidos mediante la investigación como estrategia pedagógica en la escuela, permite creación de consciencia ambiental, pero también estimula el desarrollo de habilidades y capacidades cognitivas, como pensamiento crítico, análisis de información, procesos de lectura, habilidades comunicativas, expresión oral, solución de problemas, sistematización de resultados, entre otras, que favorecen la formación cultura ciudadana, en ciencia, tecnología e innovación.

Por otro lado, para llevar a cabo la evaluación de los impactos adversos derivados de las formas de manejo integral de los residuos sólidos, se requiere en primer término definir cuáles son los contaminantes potenciales que pueden causar afectaciones en función de las propiedades que los hacen peligrosos y las condicio- nes que influyen para convertirse en un riesgo a la salud y al ambiente. En lo que se refiere a las sustancias químicas que pueden estar contenidas en los residuos o que se pueden generar como resultado de sus formas de manejo, lo que importa es conocer, si poseen características que les confieren la potencialidad de generar explosiones, incendios, corrosión de materiales, efectos tóxicos, deterioro de la calidad del aire, agua y suelo.

También se deben de conocer las posibles implicaciones para los suelos, el agua y el aire de la disposición o de los procesos que inciden o a los que se someterá la materia orgánica contenida en los residuos y, en su caso, los efectos adversos que se puedan llegar a producir en la salud humana o en los ecosistemas. Así, por ejemplo, se debe tener presente que la materia orgánica depositada en el suelo puede contribuir a la lixiviación de sustancias y a la contaminación subsecuente de los mantos freáticos; puede dar lugar también a procesos de combustión que provoquen incendios; puede generar los llamados gases de efecto invernadero asociados al cambio climático global o favorecer el desarrollo de fauna nociva (ratas, moscas, cucarachas).

Lo anterior demuestra que la investigación como estrategia pedagógica es una herramienta didáctica, que permite al docente integrar los contenidos curriculares a la praxis, impartiendo un conocimiento contextualizado, donde el estudiante mantiene un rol activo en la construcción de este conocimiento, pero que además tiene un componente social, que permite generar soluciones a realidades propias del entorno en el que este se desarrolla.

Por último, esta propuesta de investigación, pretende incentivar la integración de la investigación como estrategia pedagógica en las escuelas con la finalidad de 
brindar una educación de calidad, que permita al estudiante desarrollar habilidades científicas, para construir su conocimiento de forma didáctica y aportar al desarrollo social de su contexto, concientizando a la comunidad en general, basando criterio enfocado en la resolución de este flagelo como son el manejo de residuos sólidos.

\section{Referencias bibliográficas}

Carrato, A., \& Marval, R. (2007). Propuesta de un programa de educación ambiental para la conservación del agua y recolección de residuos sólidos, aplicable a las comunidades. Universidad del Oriente, Venezuela.

Cochran, M Y Litle, S. (2006). Más allá de la certidumbre adoptar una actitud indagadora sobre la práctica. Ed. Octaedro. Barcelona.

García, H., Toyo, L., Acosta, Y., Rodríguez, L., \& El Zauahre, M. (2015). Percepción del manejo de residuos sólidos urbanos fracción inorgánica en una comunidad universitaria. Multiciencias, Vol. 14(3).

Garmendia, A, y Dávila, A. (2006). Minimización y Manejo ambiental de los residuos sólidos. Editorial, Semarnat. México.

Hernández, R., Fernández, C., \& Baptista, P. (2010). Metodología de la investigación. México. Editorial Mc Graw Hill.

Herrera, K., \& Bravo, E. (2013). Perspectiva de la ecología en la comprensión de los comportamientos ambientales. Omnia, 19 (3), 20-30.

Mariño, G. (2010). El diálogo en la educación de jóvenes y adultos. Dos propuestas pedagógicas para implementarlo. En: El taller dialógico/la recuperación de experiencias laborales. Bogotá. OEI.
Medina, M. (2017). Reciclaje de desechos sólidos en América Latina. Frontera Norte, 11(21), 7-31.

Mejía, M., \& Manjarrés, M. (2010). La Investigación como Estrategia Pedagógica. Programa Ondas - Colciencias.

Moyano, E. (2012). Manejo de residuos en la institución educativa departamental instituto de promoción social. Liberia, viotá. Perspectivas bioéticas.

Ortega, P., Peñuela, D \& López, D. (2009). Sujetos y prácticas de la pedagogía crítica. Ediciones El Búho. Bogotá.

Picardo, O., Balmore, R., \& Escobar, J. (2004). Diccionario enciclopédico de ciencias de la educación. San Salvador: El Salvador.

Pozo, J. \& Gómez, M. (2006). Aprender y enseñar ciencia. Ediciones Morata. 5ta. Edición. Madrid.

Quiñónez Cortez, L. I. (2016). Contaminación ambiental por manejo de residuos sólidos en casa hogar infanto-juvenil femenina propuesta de un plan de manejo.

Ramírez, R. (2018). Tendencias emergentes de la gestión de talento humano en las organizaciones. Pp. 101-107.

Sáez, A., \& Urdaneta, J. (2014). Manejo de residuos sólidos en América Latina y el Caribe. Omnia, Vol. 20 (3).

Sandoval, A, \& Barrera S. (2017). Estudio de la relación costo-beneficio para la aplicación de un programa de educación ambiental de manejo de residuos sólidos en la unidad de planeación zonal 71 de la localidad de suba, en Bogotá. (Bachelor's thesis, Universidad Distrital Francisco José de Caldas). 